\title{
Interplay between glycaemic status, lipid peroxidation and hyperlipidaemia in subjects with varying glucose metabolism
}

\author{
Paul $\mathrm{R}^{1^{*}}$, Mukkadan $\mathrm{JK}^{2}$
}

*Corresponding author:

${ }^{1}$ Ms. Remya Paul, M.Sc., Research Scholar, Little Flower

Medical Research Centre, Angamaly

Email: remyapaul.k@gmail.com $\underline{\text { ORCID }}$

${ }^{2}$ Research Director, Little Flower Medical Research Centre, Angamaly.

\section{Information about the article:}

Received: Jan. 17, 2019

Accepted: Feb. 20, 2019

Published online: Dec. 27, 2019

\section{Publisher}

Nepal Health Research Society, Bahundhara -6, Gokarnesowor Municipality, Kathmandu, Nepal eISSN 2382-5545, ISSN 2676-1343 (Print)

(c) The Author(s). 2019

Content licensing: CC BY 4.0

\begin{abstract}
Background

Diabetes is one of the largest global health emergencies of the 21st century. About 425 million people worldwide or $8.8 \%$ of adults between the ages of 20-79 years are estimated to have diabetes. There are evidences which show that higher blood glucose and cholesterol levels have an association with free radical-mediated lipid peroxidation. The aim of the study was to compare and correlate glycosylated haemoglobin (HbA1c), lipid peroxidation marker malondialdehyde (MDA), lipid profile, and antioxidant enzyme superoxide dismutase (SOD) activity in subjects with varying levels of glucose metabolism.
\end{abstract}

\section{Materials and methods}

This was a cross sectional study. Based on fasting plasma glucose and HbA1c, subjects were divided into diabetic patients $(n=40)$, pre-diabetic patients $(n=38)$ and normal patients $(n=41)$. Subjects were tested for lipid profile, MDA, and SOD activity. The comparison of parameter between the groups was carried out using one-way ANOVA followed by tukey's multiple comparison test. The correlation between parameter was analyzed by Karl Pearson correlation coefficient using SPSS 20.0 .

\section{Results}

The serum MDA levels (nmol/ml) were significantly higher in pre-diabetes $(3.11 \pm 0.40)$ and diabetes mellitus (3.55 \pm 0.88$)$ compared to normal controls $(2.04 \pm 0.99)$. The SOD activity $(\mathrm{U} / \mathrm{ml})$ were lower in diabetes $(7.69 \pm 3.83)$ and pre-diabetic subjects $(8.13 \pm 2.15)$ compared to normal subjects (11.16 \pm 3.61$)$. MDA has significant positive correlation with HbA1c and total cholesterol level. SOD has significant negative correlation with $\mathrm{HbA1c}$ and Total cholesterol/High Density Lipoprotein (TC/ HDL) ratio.

\section{Conclusion}

The findings strongly confirmed that there is a continuous interplay between glycemic status, lipid peroxidation and hyperlipidemia in which one factor perpetuates another leading to the progression of disease.

\section{Keywords}

Diabetes mellitus, HbA1c, lipid peroxidation, SOD 